\title{
Experimental Investigation on Performance of Fabrics for Indirect Evaporative Cooling Applications
}

\author{
Peng $\mathrm{Xu}^{\mathrm{a}, \mathrm{b}}$, Xiaoli Ma ${ }^{\mathrm{a}}$, Xudong Zhao ${ }^{\mathrm{a}}$, Kevin S Fancy ${ }^{\mathrm{a}}$ \\ a School of Engineering, University of Hull, UK, HU6 7RX \\ ${ }^{b}$ Key lab of HVAC, Beijing University of Civil Engineering and Architecture, Beijing, China, 100044 \\ *Corresponding author: x.ma@hull.ac.uk \\ Tel: +44 (0)1482 466848; Fax: +44 (0)1482 466664
}

\begin{abstract}
Indirect evaporative cooling, by using water evaporation to absorb heat to lower the air temperature without adding moisture, is an extremely low energy and environmentally friendly cooling principle. The properties of the wet channel surface in an indirect evaporating cooler, i.e. its moisture wicking ability, diffusivity and evaporation ability, can greatly affect cooling efficiency and performance. Irregular fibres help to divert moisture and enlarge the wetted area, thus promoting evaporation. A range of fabrics (textiles) weaved from various fibres were experimentally tested and compared to Kraft paper, which has been conventionally used as a wet surface medium in evaporative coolers. It was found that most of the textile fabrics have superior properties in moisture wicking ability, diffusivity and evaporation ability. Compared with Kraft paper, the wicking ability of some fabrics was found to be $171 \%$ to $182 \%$ higher, the diffusion ability $298 \%$ to $396 \%$ higher and evaporation ability $77 \%$ to $93 \%$ higher. A general assessment concerning both the moisture transfer and mechanical properties found that two of the fabrics were most suitable for indirective evaporative cooling applications.
\end{abstract}

Key words: moisture wicking, evaporation, diffusivity, indirect evaporative cooling

\section{INTRODUCTION}


The current air conditioning market is dominated by mechanical vapour compression systems, which are energy intensive and suffer from poor thermal performance in hot climate conditions. Hence, there is a renewed interest in the use of evaporative cooling for thermal comfort in buildings [1]. This cooling method utilises the principle of water evaporation for absorbing heat existing in ambient, therefore, consuming much lower electricity compared to mechanical compression system. Direct evaporative cooling system and indirect evaporative cooling system are two types of evaporative cooling systems commonly used nowadays [2]. Direct evaporative cooling systems can lower the temperature of air using the latent heat of evaporation. As a result, warm dry air is changed into cool moist air. Indirect evaporative cooling systems have the advantage of being able to lower the air temperature without increasing humidity of the conditioned space and avoid potential health issues from contaminated water droplets entering occupied spaces (as associated with direct evaporative cooling systems) [3]. Thermodynamically, an indirect evaporative air cooler passes the primary (product) air over the dry side of a heat/mass exchanging wall, and the secondary (working) air over its opposite wet side. The wet side absorbs heat from the dry side by evaporating water and therefore cooling air from dry side, while the latent heat from vaporising water is given to air on the wet side [4]. Figure 1 shows the principle of direct and indirect evaporative cooling. As a result of the above mentioned advantage from indirect evaporative cooling, its use has recently become widespread in evaporative air conditioning.

The evaporative air cooler is sample and compact. A typical indirect evaporative cooler is constructed of a heat and mass exchanger, fans, a water distributor/a water basin and a small water pump. Usually all the components can be accommodated into a cuboid envelop (casing) 
with an air inlet (for fresh air intaking) and two air outlets (exhaust air outlet and product air outlet respectively). The cooler can be integrated in buildings very easily, e.g. putting in an utility room in the house, in any room closing to a wall, on the roof of a buildings, or outside the buildings attached to a wall. All we need to do is to connect the air inlet to the outdoor fresh air, exhaust air outlet to outside buildings and product air outlet to the rooms to be cooled through ducts, which may need to make appropriate holes in the wall or/and ceiling of the buildings.

Researches studies into improving the performance of indirect evaporative cooling are numerous. In addition to developing new types of heat and mass exchangers which aims to enhance the heat and mass transfer rates by optimsing the geometry of the heat and mass exchangers $[2,3,5-7]$, research has also focused on the heat and mass exchanger medium (wet surface material) [8-15]. This is because the properties of the heat/mass exchanging medium, i.e. its moisture wicking ability, diffusivity and evaporation ability, can greatly affect the the wetted area as well as the moisture diffusion and evaporation rate, and therefore affect cooling efficiency and performance of evaporative cooling systems.

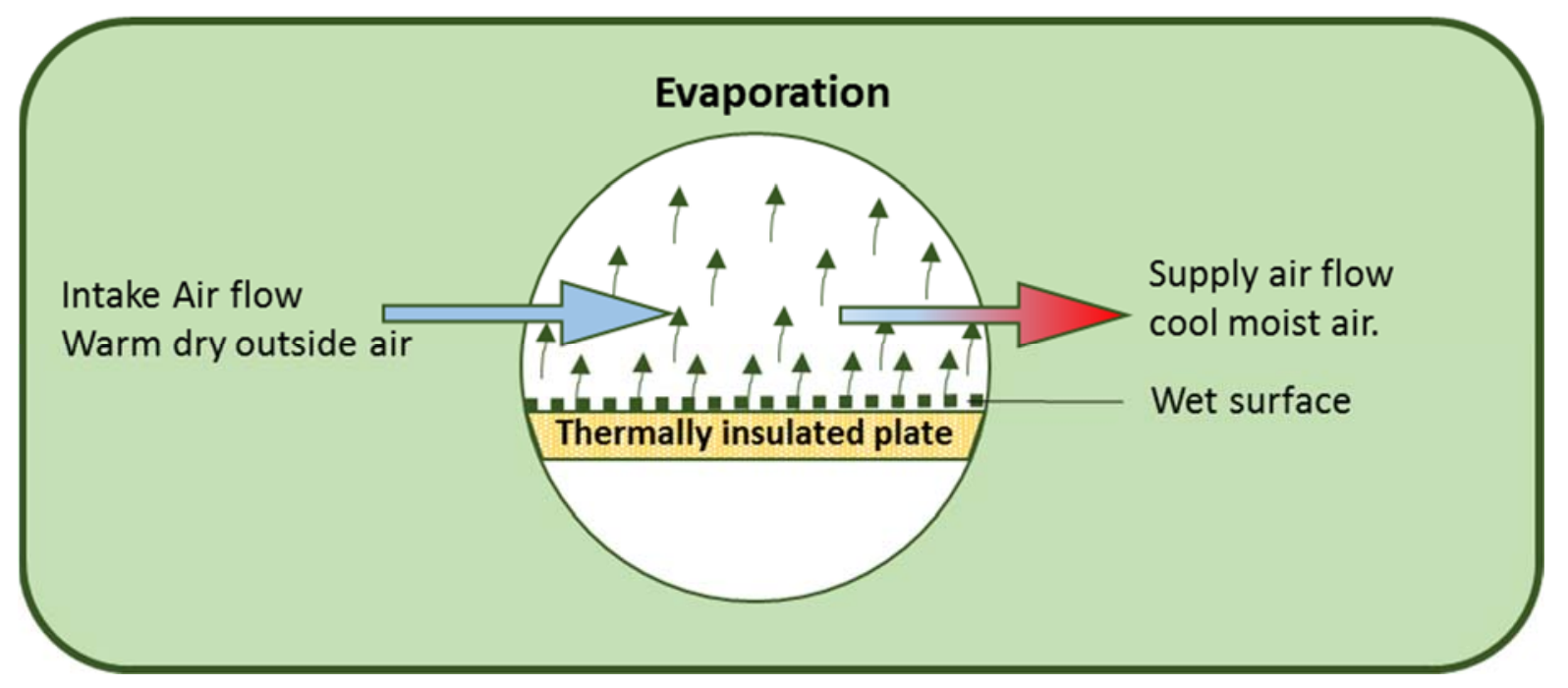




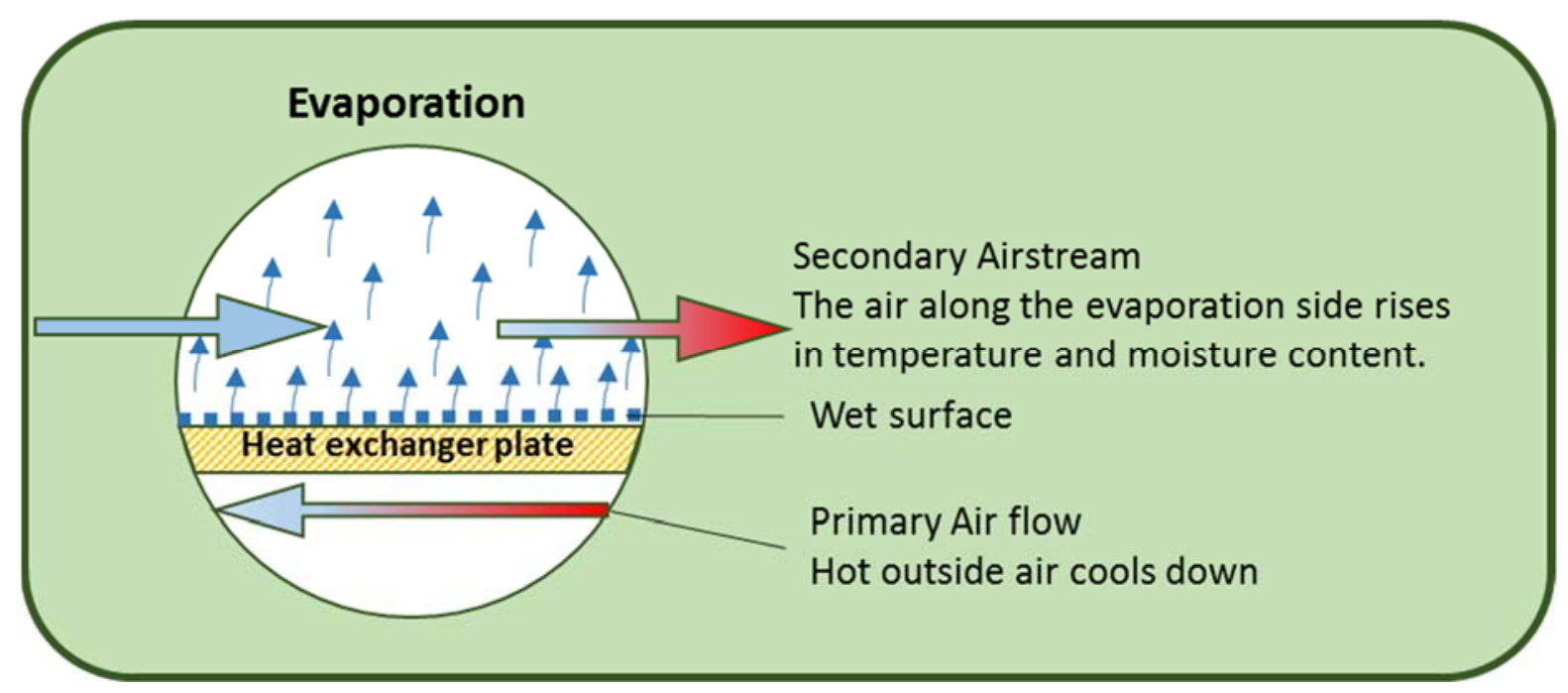

(b)

Figure 1 Principle of evaporative cooling: (a) direct, (b) indirect

Bruno [8] constructed a flat-plate cross-flow heat and mass exchanger, which used a special medium with high water retention and wickability characteristics as the wet channel, and a moisture-impervious membrane as the dry channel. Tests indicated that the exchanger had a dew point effectiveness of $75 \%$, which was comparatively low under the given operational condition.

Velasco et al [9] carried out an experimental study into a polycarbonate based indirect evaporative cooling heat exchanger, which is benefited from low cost and weight, with no corrosion problems; the limitations caused by low thermal conductivity were reduced by minimising plate thickness. The results showed that higher outdoor air temperatures or air flow rates facilitated in obtain enhancing cooling performance of the system.

A wide range of materials have been commonly used as the heat/mass exchanging medium, and may be classified as metal, fibre, ceramic, zeolite and carbon types. Metal types include metal 
forms, metal wools, sintered metals and wicked metal plates/tubes. Fibre types include paper board, cloth (wood or glass) fibres. Ceramic types includes silicon carbide/silicon carbide composites, zirconia ceramics, zirconia-toughened aluminium, zirconium dioxide, aluminium oxide, and aluminium nitride and polystyrene composites. Zeolite types includes porous ceramics, molecular sieves and synthetic polymers, and carbon fibre refers to carbon-carbon composites and activated carbon. Zhao et al [10] conducted a comparative study on the above types of materials as potential heat and mass transfer media used in indirect evaporative cooling systems, and the results showed that thermal properties of the materials, i.e., thermal conductivity and water-retaining capacity (porosity), had little impact on system heat/mass transfer, and therefore, these two parameters had low key roles in terms of material selection. Instead, shape formation/holding ability, durability, compatibility with water-proof coatings, contamination risk as well as cost, are more important concerns in this regard.

Some natural fibers have been investigated for their evaporating cooling effect [11-14].

Maurya et al [11] comparatively studied three types of cooling pad made of a aspen fiber, cellulose, and coconut coir, based on weather conditions of Bhopal, India in summer. The results showed that the saturated efficiency at an air velocity $0.5 \mathrm{~m} / \mathrm{s}$ was highest for aspen material at $80.99 \%$ compared to $69.58 \%$ for the cellulose and $68.15 \%$ for the coconut coir. The saturation efficiency of the pad depends upon the wetted surface area of the pad because a higher wetted surface area causes greater evaporation of water into air, thereby decreasing the air temperature. The performance of coconut coir is also satisfactory; it has good water soaking ability, and its performance characteristic are very close to the cellulose pad: it may become a viable alternative. 
Kulkarni et al [12] reported a theoretical performance study of an evaporative cooler with different cooling pad shapes and materials. The performance of rectangular, cylindrical, and hexagonal shapes of evaporative cooler pads, based on rigid cellulose, aspen, corrugated paper, and high density polyethylene (HDPE) were theoretically analyzed. It was found that the highest saturation efficiency was $91 \%$ for the hexagonal pad of aspen material and the lowest value of $72.4 \%$ was obtained for the rectangular pad of cellulose material under the simulated working conditions.

Soponpongpipat [13] studied the evaporative cooling efficiency and pressure drop of recycled HDPE and rice husk as wetted pads in an evaporative cooling system. A commercial wetted pad was also tested in order to compare results with the rice husk and recycled HDPE pads. It was found that the rice husk wetted pad gave an average saturation efficiency of $55.9 \%$, while HDPE gave the average saturation efficiency of $29.1 \%$ for the testing conditions. However, the pressure drop across the rice husk and recycled HDPE pads was significantly higher than that of the commercial wetted pad.

Faleh [14] studied the cooling performance of an evaporate cooler by using cooling pads made of different materials such as jute, luffa and palm fiber. As a reference, a widely used commercial Aspen-wood excelsior wetted pad is evaluated as well. The majority of the commercially available wetted surfaces are wood based. Salt deposition and mold forming usually clog these surfaces. The experiment was set with a flow velocity through the cooling pads at $2.4 \mathrm{~m} / \mathrm{s}$. In conclusion, the study showed cooling efficiency of the cooling pads made of various materials as follows: $62.1 \%$ for jute, $55.1 \%$ for luffa, $49.5 \%$ for the commercial cooling pad and $38.9 \%$ for palm fiber. The results from the cooling efficiency degradation 
indicated that luffa had an overall advantage over the other fibers. Palm fibers and the commercial material demonstrated a significant reduction in the cooling efficiency, while jute showed the highest deterioration. However, if the jute surface could be treated to offer higher mold resistance characteristics, it would provide the best alternative.

Cloth fabrics, as common commercial available materials, are highly durable, exhibit a low pressure drop, can be easily shaped into various geometries, easily cleaned and replaced. Also, they are able to avoid bacterial growth when used as wet surfaces and exhibit larger capillary force; hence they are potentially highly effective materials for evaporative cooling. However, relatively few reports can be found where this type of materials have been investigated for evaporative cooling applications. Niyomvasa et al [15] parformed comparative studies of two types of cooling pad made from a curtain fabric and a raw cotton fabric by experiment. They showed that the average difference in temperatures between inlet and outlet were $2.9^{\circ} \mathrm{C}$ and $1.7^{\circ} \mathrm{C}$ for the curtain and raw cotton fabrics, respectively. The average saturation efficiency was found to be $54.8 \%$ for the curtain fabric, and $33.2 \%$ for the raw cotton fabric.

Cloth fabrics have are inherently thin, resulting in higher heat transfer, which allows a large amount of heat to be conducted from the dry side of the wall to the wet side; thus they are particularly suitable for indirect cooling applications. As cloth fabrics are not rigid, they must be attached to rigid sheet to construct a wet pad. For an indirect evaporative cooling system, the cloth fabrics need to be attached onto one side of rigid sheet with high thermal conductivity, such as aluminium, to form a wet side and the opposite side of the assembly then performs as the dry side. This paper presents an experimental study of the performance of six different cloth 
fabrics samples weaved from various fibres and one Kraft paper sample in terms of their moisture wicking, diffusivity rate and evaporation rate; the results are compared to enable the optimum fabrics for indirect evaporating cooling to be identified.

\section{MOISTURE TRANSFER PROPERTIES OF FABRICS AND THEIR EVALUATION}

Evaporative cooling is a thermodynamic process. The evaporative cooling performance depends on the pad characteristics and mass flow rate of air. When air passes through porous wet media, a proportion of water evaporates and reduces the air temperature. The efficiency of an evaporative cooling system is directly affected by factors such as the type of wet surface material, surface area, mass flow rates of air, pad efficiency, dryness of the air and RH of air passing through the wet channels and volume of water used. Hence selection of wet surface media requires careful attention.

The major concerns of a wet surface medium for evaporative cooling are moisture wicking ability and evaporation ability. The moisture wicking ability refers to movement of moisture within a fabric by capillary action, usually along the filament surface, to where it can evaporate quickly. Wicking ability is commonly evaluated by the vertical wicking and diffusivity (horizontal wicking) abilities. A higher wicking ability can divert moisture and enlarge the wetted area, thereby maximising the effective wet surface which promotes evaporation in the cooler. Evaporation ability is evaluated by evaporation rate of moisture from the wet surface. This was carried out by dropping a certain amount of water on the fabric surface, after the water fully spread, observing and recording the wetted area reduction process. The amount of the moisture on the fabric evaporating to ambient at a certain time interval can be calculated from 
the wetted area reduction process, the unit of the measurement is microgram per second $(\mathrm{mg} / \mathrm{s})$. More details of the method for measuring and evaluating the evaporation rate are presented in section 3.2. The moisture evaporation rate from the wet surface directly affects evaporative cooling efficiency. Therefore, a higher wicking ability and evaporation rate can greatly increase the performance of an evaporative cooler.

Wicking is the spontaneous flow of a liquid within a porous substance, driven by capillary forces. Capillarity is the ability of liquids to penetrate into fine pores with wettable walls and be displaced from those with non-wettable walls [16]. Capillary rise in fibrous structures is frequently observed in many fields, such as wetting and wicking in textiles, paper and porous media [17]. Capillary wetting is attributed to the meniscus formed by fibres and yarns in warp and weft directions, especially in the interstitial area [18]. Wicking is determined mainly by effective capillary pore distribution, pathways and surface tension, whereas the evaporation rate of a material is related to the macromolecular structure of the fibre [19]. In many research studies, textile yarns were treated either as porous media [20-22], where the liquid transport is described by Darcy's law [23], or as capillary tubes [24-27] in which the liquid which can be modelled by Lucas-Washburn kinetics[28]. Although these theories can be applied, in the first case, the characteristic parameters, such as permeability, are difficult to quantify and are obtained empirically; in the second case, the effective radius of the capillary tube, the effective contact angle, and so forth are determined by fitting experimental data [29]. Many researchers have tried to find the moisture transfer properties of textiles by objectively using different test methods. Kissa [30] has found that assuming the fabric was impermeable to the liquid, the spreading rate could be represented by the following equation: 


$$
A=K(\gamma / \eta)^{u} V^{m} t^{n}
$$

Where, $A$ is the area of the liquid drop at time $t, V$ is the volume of the drop, $\gamma$ is the surface tension, $\eta$ is the viscosity, and $K$ the capillary sorption coefficient. All the above studies showed that moisture transfer properties of the textile fabrics are affected by characteristics such as the fibre material, their fibrous structures, warp and weft directions, surface tension, viscosity and macromolecular structure.

In this work, experimental lab tests for a range of different cloth fabrics were carried out to identify their wicking and evaporation abilities and mechanical suitability for indirective evaporative cooling applications. A Kraft paper sample, which is conventionally used as a wet medium in evaporative cooling, was also tested for comparative purpose.

The moisture wicking ability was evaluated by wicking height rate, and the diffusivity and evaporation ability was evaluated by diffusivity rate and evaporation rate, defined as follows:

Wicking height rate is expressed by:

$$
W=\frac{\Delta h}{\Delta t_{1}}
$$

Where, $W$ is the vertical wicking rate $(\mathrm{cm} / \mathrm{min})$ and $\Delta h$ is wicking height increase $(\mathrm{cm})$ at a designated time interval $\Delta t_{1}(\mathrm{~min})$.

The diffusivity rate is expressed by:

$$
D=\frac{\Delta A}{\Delta t}
$$


Where, $D$ is the diffusivity rate $\left(\mathrm{cm}^{2} / \mathrm{s}\right)$ and $\Delta A$ is wetted area increase $\left(\mathrm{cm}^{2}\right)$ at a designated time interval $\Delta t(\mathrm{sec})$.

The evaporation rate is expressed by:

$$
E=\frac{\Delta M}{\Delta t}
$$

Where, $E$ is the evaporation rate $(\mathrm{mg} / \mathrm{s})$ and $\Delta M$ is the amount moisture on the fabric evaporating to ambient $(\mathrm{mg})$ at a designated time interval $\Delta t(\mathrm{sec})$.

\section{PERFORMANCE TESTING OF THE FABRICS}

Performance tests of six different cloth fabric samples and one Kraft paper sample was carried out to identify their vertical moisture wicking ability, diffusivity ability, evaporation ability and mechnical suitabiliy. The Kraft paper, which has been conventionally used as a wet surface material in evaporative cooler was tested for comparison of its performance to that of clothing fabrics.

The six cloth fabrics are all commercial available athletic clothing materials. The athletic clothing materials are performance fabrics that have good wicking ability and evaporation ability to keep the body dry [31].

\subsection{Specifications of the Tested Fabrics}

The specifications of the tested fabrics are listed in Table 1. Table 1 lists the images, fabric brand, weaving, weight and breadth of each fabric (sample numbers are assigned to each sample for convenience of description in other parts of the paper). The difference between the fabrics 
is determined by "Fabric brand" which indicates the material used to weave the fabric and "Weaving" which indicates the weaving method. Both sides of each fabric are shown in each image if they are different. The costs of the textile fabrics for their minimum purchase are listed in Table 2.

Table 1 Specifications of the fabrics

\begin{tabular}{|c|c|c|c|c|c|}
\hline \multirow{2}{*}{$\begin{array}{c}\text { Sample } \\
\text { No. }\end{array}$} & \multirow{2}{*}{ Photo of the fabric } & \multicolumn{4}{|c|}{ Specification } \\
\hline & & Fabric brand & Weaving & Weight $\left(\mathrm{g} / \mathrm{m}^{2}\right)$ & Breadth $(\mathrm{cm})$ \\
\hline 1 & & Coolpass ${ }^{\circledR}$ & $\begin{array}{l}\text { bird eye mesh fabric } \\
\qquad 100 D^{\star} 72 F\end{array}$ & 170 & 180 \\
\hline 2 & & Kraft paper & - & 117 & 32 \\
\hline 3 & 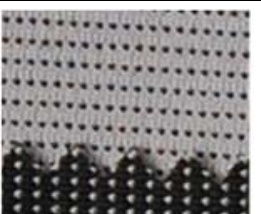 & $\begin{array}{c}\text { Bamboo charcoal } \\
+ \\
\text { Coolmax }{ }^{\circledR} \text { active }\end{array}$ & $75 \mathrm{D}$ & 150 & 155 \\
\hline 4 & & Coolpass ${ }^{\circledR}$ & $\begin{array}{c}\text { knitted (double) pique } \\
\text { mesh } \\
75 D^{\star} 72 \mathrm{~F}\end{array}$ & 150 & 170 \\
\hline 5 & & Topcool® spandex & $\begin{array}{c}\text { single jersey } \\
88 / 12\end{array}$ & 170 & 165 \\
\hline 6 & & $\begin{array}{c}320 D \\
\text { Supplex®+3M® }\end{array}$ & $70 D^{*}(160 D+160 D)$ & 130 & 150 \\
\hline
\end{tabular}




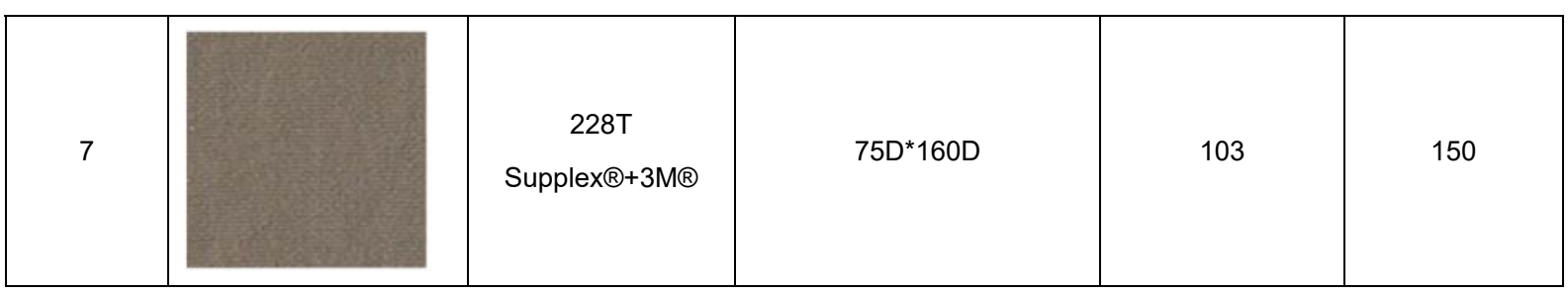

Table 2 Costs of the textile fabrics

\begin{tabular}{|l|c|c|c|c|c|c|}
\hline Sample No. & $\mathbf{1}$ & $\mathbf{3}$ & $\mathbf{4}$ & $\mathbf{5}$ & $\mathbf{6}$ & $\mathbf{7}$ \\
\hline $\begin{array}{l}\text { Cost per } \\
\text { kilogram in } \\
\begin{array}{l}\text { Chinese currency } \\
\text { (Yuan) }\end{array}\end{array}$ & $¥ 36 / \mathrm{kg}$ & $¥ 75 / \mathrm{kg}$ & $¥ 30 / \mathrm{kg}$ & $¥ 50 / \mathrm{kg}$ & $¥ 19.5 / \mathrm{m}$ & $¥ 17.5 / \mathrm{m}$ \\
\hline Minimum order & $200 \mathrm{~kg}$ & $1000 \mathrm{~kg}$ & $200 \mathrm{~kg}$ & $500 \mathrm{~kg}$ & $1000 \mathrm{~m}$ & $1000 \mathrm{~m}$ \\
\hline
\end{tabular}

\subsection{Experimental testing rig and testing methodology}

Figure 2 shows the testing rig, in which major parts include a high resolution $(1920 \times 1080$ pixels $)$ camera, a ruler, a burette with drop flow controller, a hygrothermograph and computer. The high resolution Logitech HD Pro webcam c910 with Carl Zeiss optics autofocus facility camera, is used to measure the dynamic movement of the liquid over the fabric surface. The camera has the facility to produce high resolution images from high-definition 10-megapixel video capture quality and automatic brightness adjustment and colour compensation. The camera is mounted on a support stand, equipped with a LED light, and connected to the computer via its USB port. The distance between the camera and fabric sample was maintained at a fixed distance.

Figure 2(a) shows the moisture vertical wicking testing. Here, the seven different fabric strips of $20 \mathrm{~cm} \times 2 \mathrm{~cm}$ were suspended vertically with their lower ends $(2.5 \mathrm{~cm})$ immersed in a reservoir of water. A ruler adjacent to the strips was used to measure the wicking height.To track the movement of water at a regular time interval, a video for the wicking process was taken with 
the camera. The video shown in the computer can enable the variation of wicking height with time to be assessed, by stopping the video at a certain time for image capture. The camera was compatible with image analysis software, hence the captured image was processed with Adobe Photoshop, which facilitated measurement of the wicking height from the ruler within the image.

Figure 2(b) shows the moisture diffusivity/evaporation testing. The fabric $(15 \mathrm{~cm} \times 15 \mathrm{~cm})$ was laid on a flat metal surface horizontally, the flat surface being fixed to a vibration-free bench. Ten water drops $(0.5 \mathrm{ml}$ of water in total) was dropped onto the centre of the fabric surface using a burette with drop flow controller, which was positioned $5 \mathrm{~mm}$ above the surface of the fabric. The moisture diffusivity/evaporation process together with a reference area of $2 \mathrm{~cm} \times 2 \mathrm{~cm}$ was taken as a video with the camera. Figure 3 shows an image of the moisture diffusivity/evaporation of Kraft paper with the reference area. The video fed to the computer enabled the moisture spread and evaporation processes to be assessed, which could be done by stopping the video at a certain time and capturing the image. Photoshop software was used to process the image to determine the pixel count of both the wetted region and reference areas, which could then be compared to determine the area of the wetted region of the fabric [32]. A schematic diagram of the commercial image processing method is shown in Figure 4 . The ambient humidity and temperature were monitored using a hygrothermograph during the testing period, shown in Figure 2(a). The testing was carried out in a laboratory environment with an ambient temperature of $22^{\circ} \mathrm{C}-24^{\circ} \mathrm{C}$ and humidity of $36 \%-40 \%$. The repeated tests showed that the effect of this small variation of the ambient condition on the wicking and evaporation performance can be neglected. 


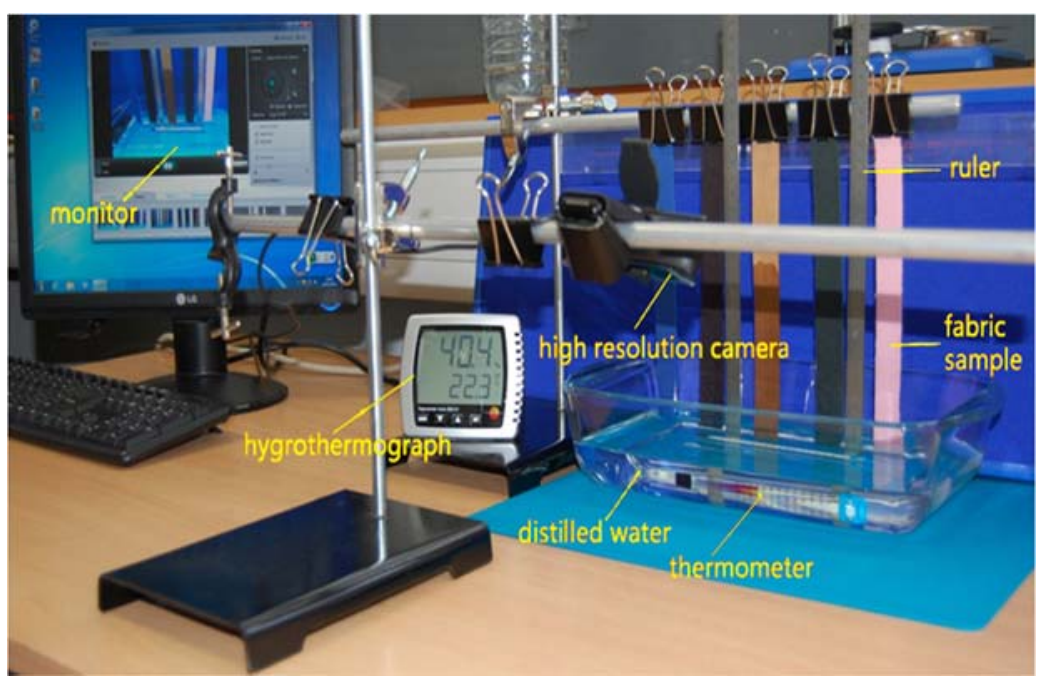

(a)

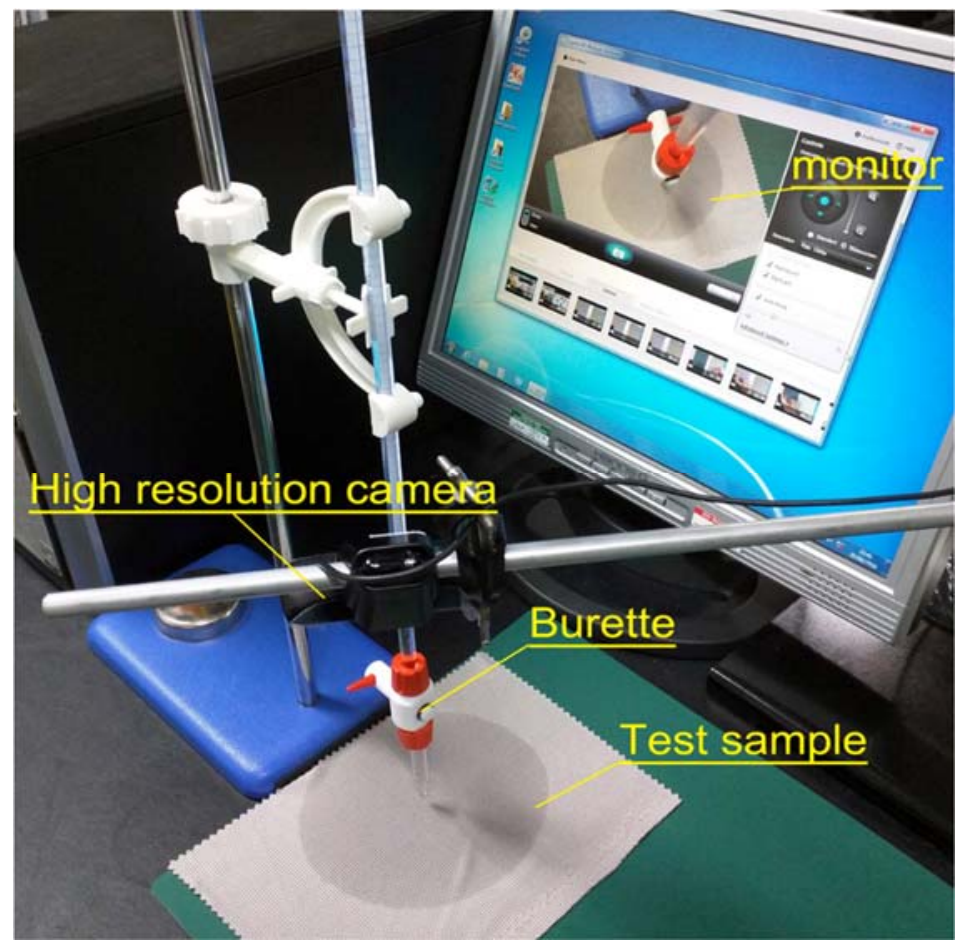

(b)

Figure 2 Experimental testing rig set up: (a)moisture wicking testing, (b) diffusivity and evaporation testing 


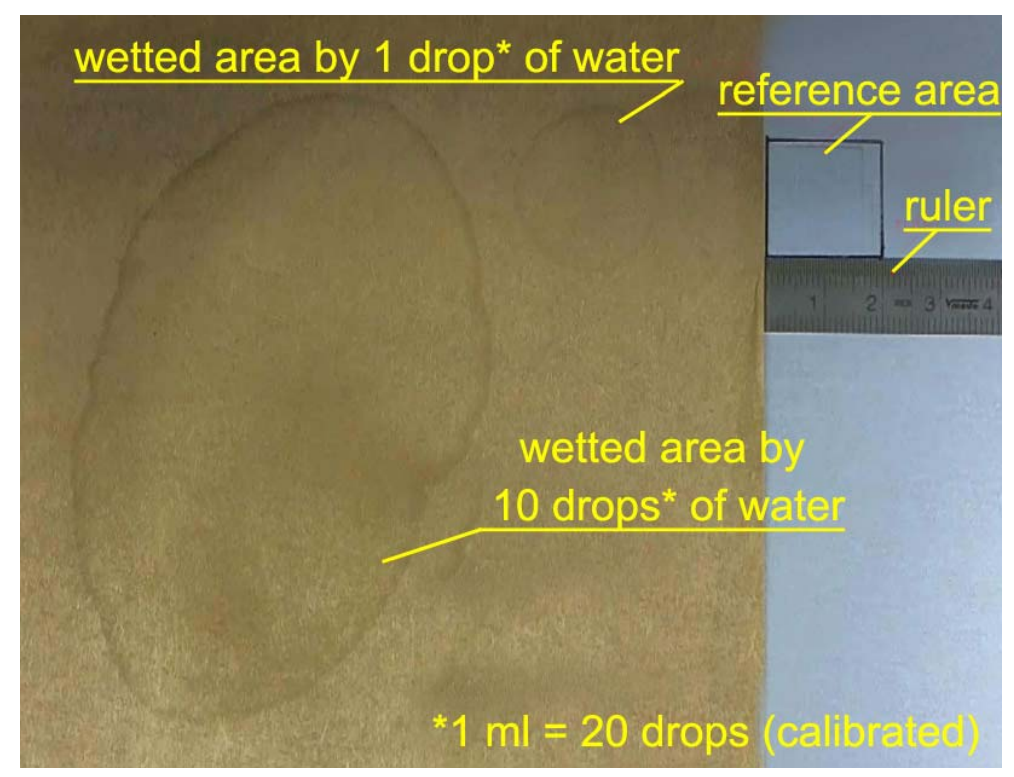

Figure 3. Kraft paper wetted area compared with the reference area

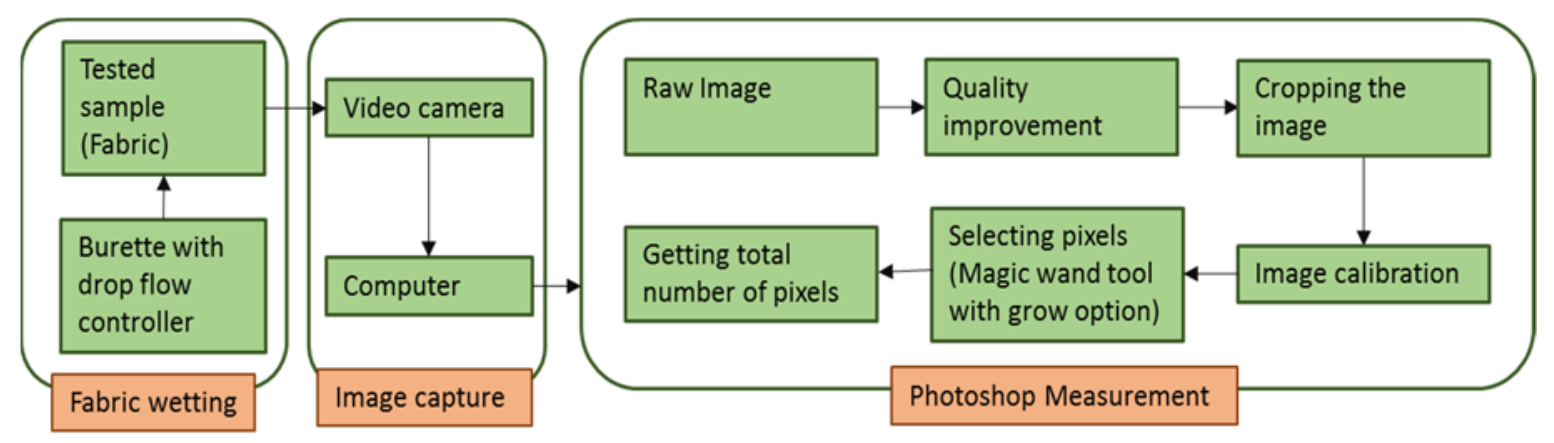

Figure 4. Schematic diagram of the commercial image processing method

Before starting a new test, the position of the camera height from the fabric surface was calibrated. For this a square $(2 \mathrm{~cm} \times 2 \mathrm{~cm})$ reference area $\left(\right.$ i.e. $\left.4 \mathrm{~cm}^{2}\right)$ was drawn on white paper and it was placed adjacent to the fabric in the region where the camera captured the image. The captured image was transferred into Photoshop and the image resolution was changed into 15 pixels $/ \mathrm{cm}$. Then the area of the square was found in terms of pixels. The total pixels were compared with the calculated value $\left(4 \mathrm{~cm}^{2}\right)$, and the camera height was repeatedly adjusted to produce new images until the total Photoshop count method result matched with the calculated result. 
Details of the Photoshop measurement were as follows: the required raw image (Figure 3) was opened to find the area in the image processing software. The image quality was adjusted by correcting brightness and contrast. Unwanted areas were removed by cropping the image. The image was then calibrated by changing the image resolution value into 15 pixels $/ \mathrm{cm}$, which is clear enough to view the irregular periphery of the image. The darken pixels (water spread area) was selected using the magic wand selection tool, assisted with the tolerance properties facility. The magic wand selection tool from the tool box is taken and clicked over the water spread area and then grow option is used from select menu for further exact selection of water spread area. After exact selection of water spread area, the histogram option was used to determine the number of pixels in the selected (water spread) area.

\section{RESULTS AND DISCUSSIONS}

\subsection{Wicking height}

The wicking height tests were carried out for 15 minutes. The results are listed in Table 3 and shown in Figures 5-7. Figure 5 shows variation of the wicking height with time for the seven different fabric samples. It is seen that the wicking heights of Samples 1, 3 and 4 increased faster and demonstrated higher vertical wicking ability than Samples 2, 5, 6, and 7. Sample 2 (Kraft paper), showed the lowest vertical wicking ability.

Table 3 Wicking height variation with time

\begin{tabular}{|c|c|c|c|c|c|c|}
\hline \multirow{2}{*}{$\begin{array}{c}\text { Sample } \\
\text { No. }\end{array}$} & \multicolumn{7}{|c|}{ Wicking height, $\mathrm{cm}$} \\
\cline { 2 - 7 } & 0.5 minute & 1 minute & 3 minutes & 5 minutes & 10 minutes & 15 minutes \\
\hline 1 & 6.1 & 7.5 & 10.7 & 12.7 & 15.8 & 17.5 \\
\hline 2 & 1.0 & 1.5 & 3.1 & 3.6 & 5.2 & 6.2 \\
\hline 3 & 5.5 & 6.9 & 9.9 & 11.8 & 14.9 & 16.8 \\
\hline 4 & 4.3 & 5.7 & 8.6 & 10.3 & 13.5 & 15.9 \\
\hline 5 & 3.7 & 4.3 & 5.5 & 6.3 & 7.1 & 7.6 \\
\hline 6 & 3.6 & 4.0 & 5.2 & 6.3 & 7.3 & 7.7 \\
\hline 7 & 1.9 & 2.5 & 4.2 & 5.2 & 6.1 & 6.6 \\
\hline
\end{tabular}




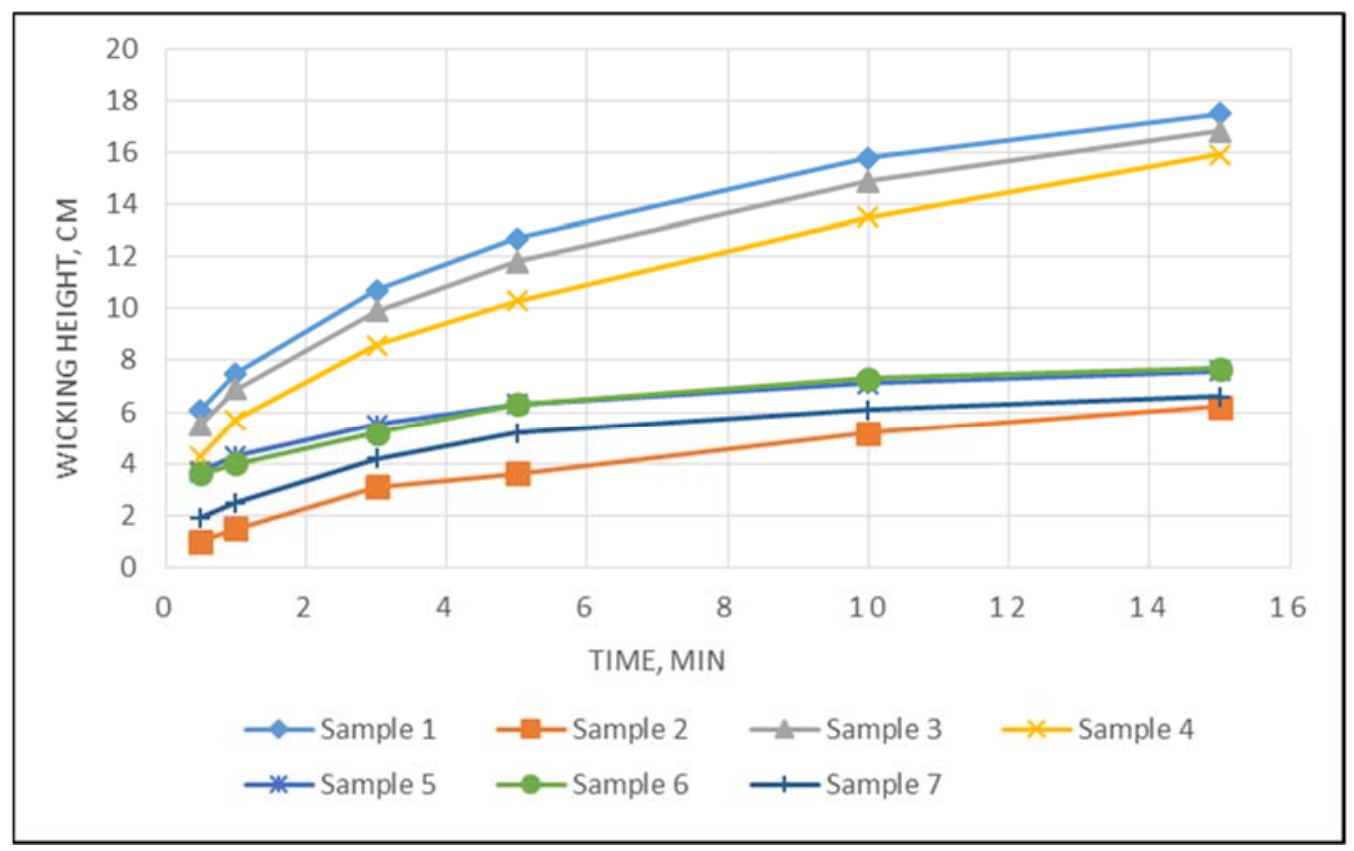

Figure 5 Variation of the wicking height with time

Figure 6 shows variation of the wicking height growth rate with time. It is seen that Samples 1, 3, and 4 performed generally higher wicking height rate than Sample 2,5,6,7. For all the samples the wicking height growing rate reduced quickly in the first three minutes, and the growth rate continued to reduce with time. Figure 6 shows that the wicking height growth rates of Sampls 2,5,6 and 7 were close to zero at the end of the testing, which indicated that their wicking heights had almost reached their highest positions in 15 minutes. However, the growth rates of Samples 1,3 and 4 were still at a lower rates of $0.34 \mathrm{~cm} / \mathrm{min}, 0.38 \mathrm{~cm} / \mathrm{min}$ and $0.48 \mathrm{~cm} / \mathrm{min}$ respectively in 15 minutes. 


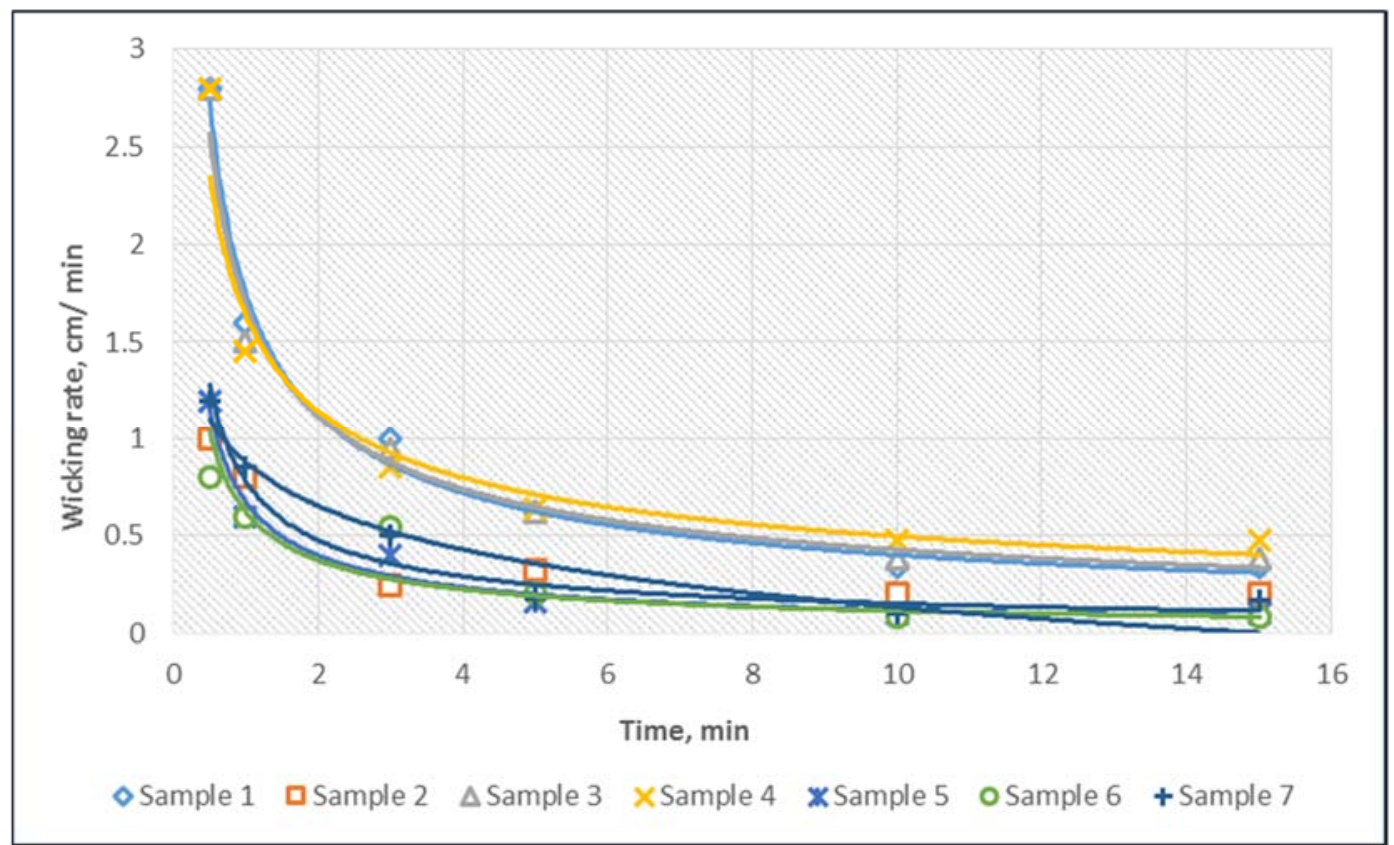

Figure 6 Variation of wicking height growing rate with time

The comparison of wicking heights at 15 minutes for the seven fabrics is shown in Figure 7. It is seen that Samples 1, 3 and 4 achieved the highest wicking heights of $17.5 \mathrm{~cm}, 16.8 \mathrm{~cm}$ and $15.9 \mathrm{~cm}$ respectively and the lowest height of $6.2 \mathrm{~cm}$ was observed with Sample 2 (Kraft paper). In summary, the testing demonstrated that all the cloth fabrics give better wicking heights when compared with the conventionally used Kraft paper and Samples 1,3 and 4 show excellent wicking height performace among the tested cloth fabrics.

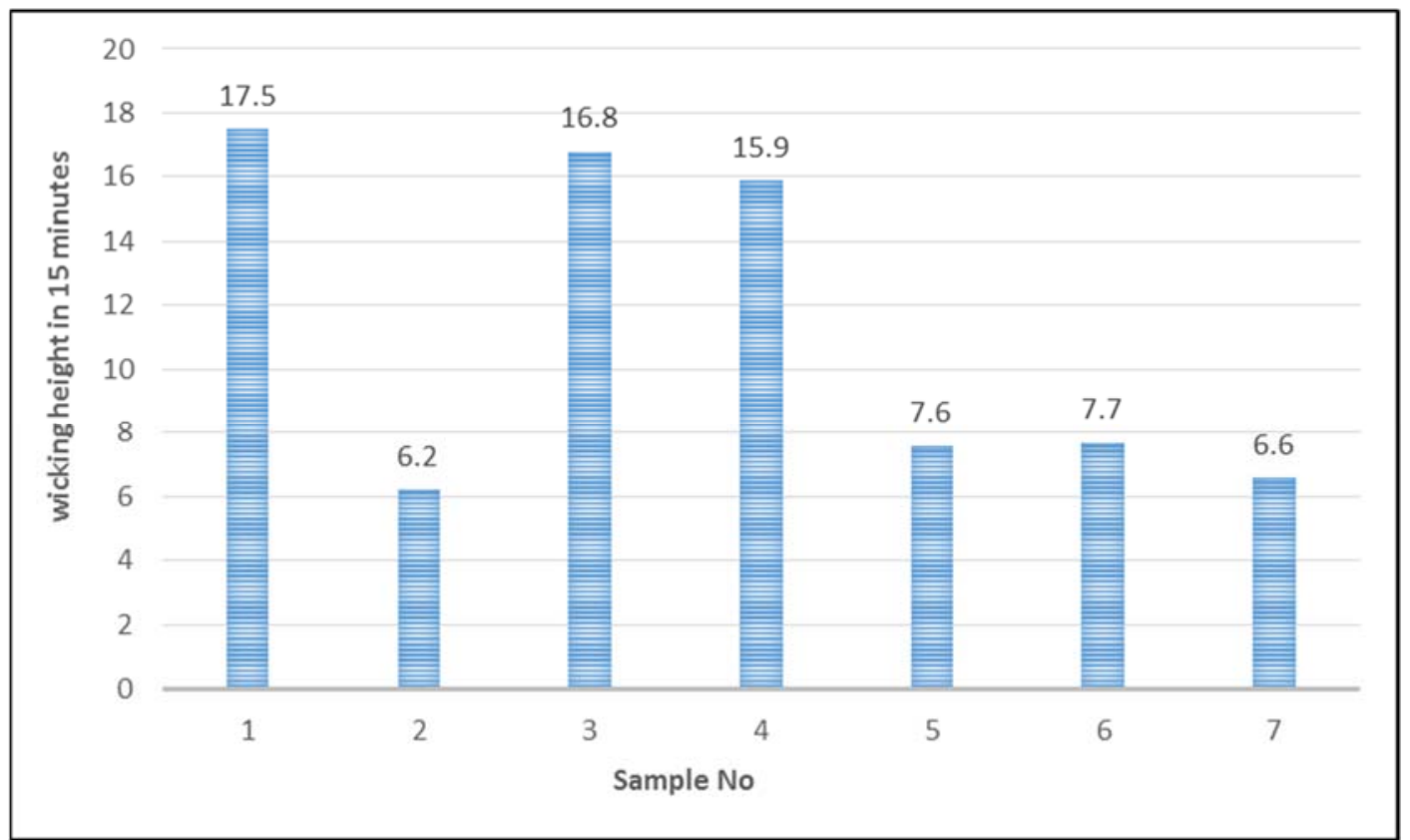




\section{Figure 7 Wicking height at 15 minutes}

\subsection{Diffusivity and evaporation ability}

An initial evaluation of moisture diffusivity/evaporation characteristics was carried out by visual assessment for each of the fabrics. Water drops (total of $0.5 \mathrm{ml}$ ) were applied onto each fabric using the burette positioned horizontally on a flat surface. It was observed that unlike other samples in which the water drops could naturally diffuse immediately (within 1 second) the drops on Samples 4 and 5 tended to retain their droplet shapes and did not appear to diffuse within in a few seconds, (see Figure 8); although they diffused eventually. Usually, the wet surfaces in an indirective evaporative cooler are positioned vertically, thus if droplets do not diffuse immediately, they would be subjected to gravitational forces, could cause the upper part of the wet surface to dry out. Therefore, Samples 4 and 5 were not considered to be a good choice for evaporative coolers, and no further diffusivity/evaporation tests were conducted for these fabric samples.

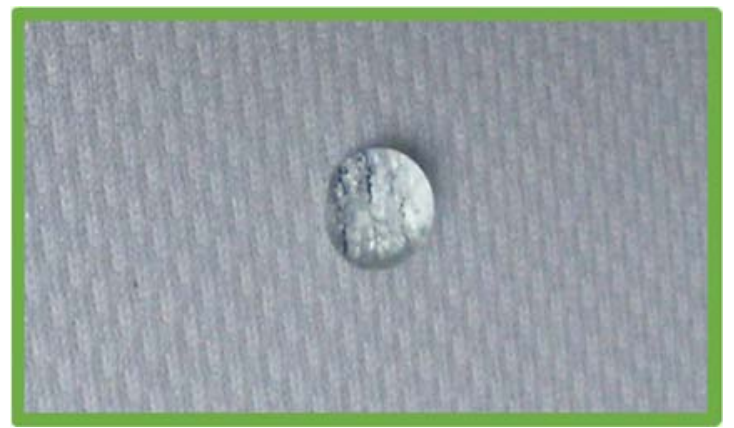

Figure 8 No diffusion within a few seconds

Testing for the remaining Samples, i.e, Samples 1, 2, 3, 6 and 7, was performed with 10 drops of water (total $0.5 \mathrm{ml}$ ) dropped on the fabric laid horizontally on a flat surface using the burette. The results are listed in Table 4 and shown in Figures 9 to 11. Table 3 lists the final wetted area 
related diffusion time and evaporation time for each sample. The evaporation time for each sample was the time required for the wet area to become completed dry, based on direct observation. This process was observed as the wetted area got smaller and smaller, and then disappeared.

Table 4 Moisture diffusion and evaporation testing results

\begin{tabular}{|c|c|c|c|c|c|}
\hline Sample No & Wetted area, $\mathrm{cm}^{2}$ & Diffusion time, $\mathrm{s}$ & $\begin{array}{l}\text { Average diffusion rate, } \\
\mathrm{cm}^{2} / \mathrm{s}\end{array}$ & $\begin{array}{l}\text { Evaporation time, } \\
\mathrm{s}\end{array}$ & $\begin{array}{l}\text { Average evaporation } \\
\text { rate, } \mathrm{mg} / \mathrm{s}\end{array}$ \\
\hline 1 & 24.3 & 210 & 0.116 & 2330 & 0.215 \\
\hline 2 & 57.3 & 560 & 0.102 & 2800 & 0.179 \\
\hline 3 & 37.5 & 268 & 0.140 & 2450 & 0.204 \\
\hline 6 & 126.6 & 250 & 0.506 & 1580 & 0.316 \\
\hline 7 & 152.3 & 375 & 0.406 & 1450 & 0.345 \\
\hline
\end{tabular}

Figure 9 shows the final wetted area on each fabric resulting from the diffusion process. It is seen that for the same amount of the water drops (total $0.5 \mathrm{ml}$ ) the final wetted areas of the tested samples are different and this can be attributed to difference in sample thickness. Thicker materials absorbed more water, resulting in a reduced area of spread. Since thinner materials can achieve larger wetted areas, this can promote more efficient evaporation when water is sprayed on the wet surfaces in an evaporative cooler. As shown in Figure 9, Samples 6 and 7 achieved larger wet areas of $126.6 \mathrm{~cm}^{2}$ and $152.3 \mathrm{~cm}^{2}$ respectively. Samples 1,2 and 3 achieved smaller wetted area of $24.3 \mathrm{~cm}^{2}, 57.3 \mathrm{~cm}^{2}$ and $37.5 \mathrm{~cm}^{2}$ repectively. 


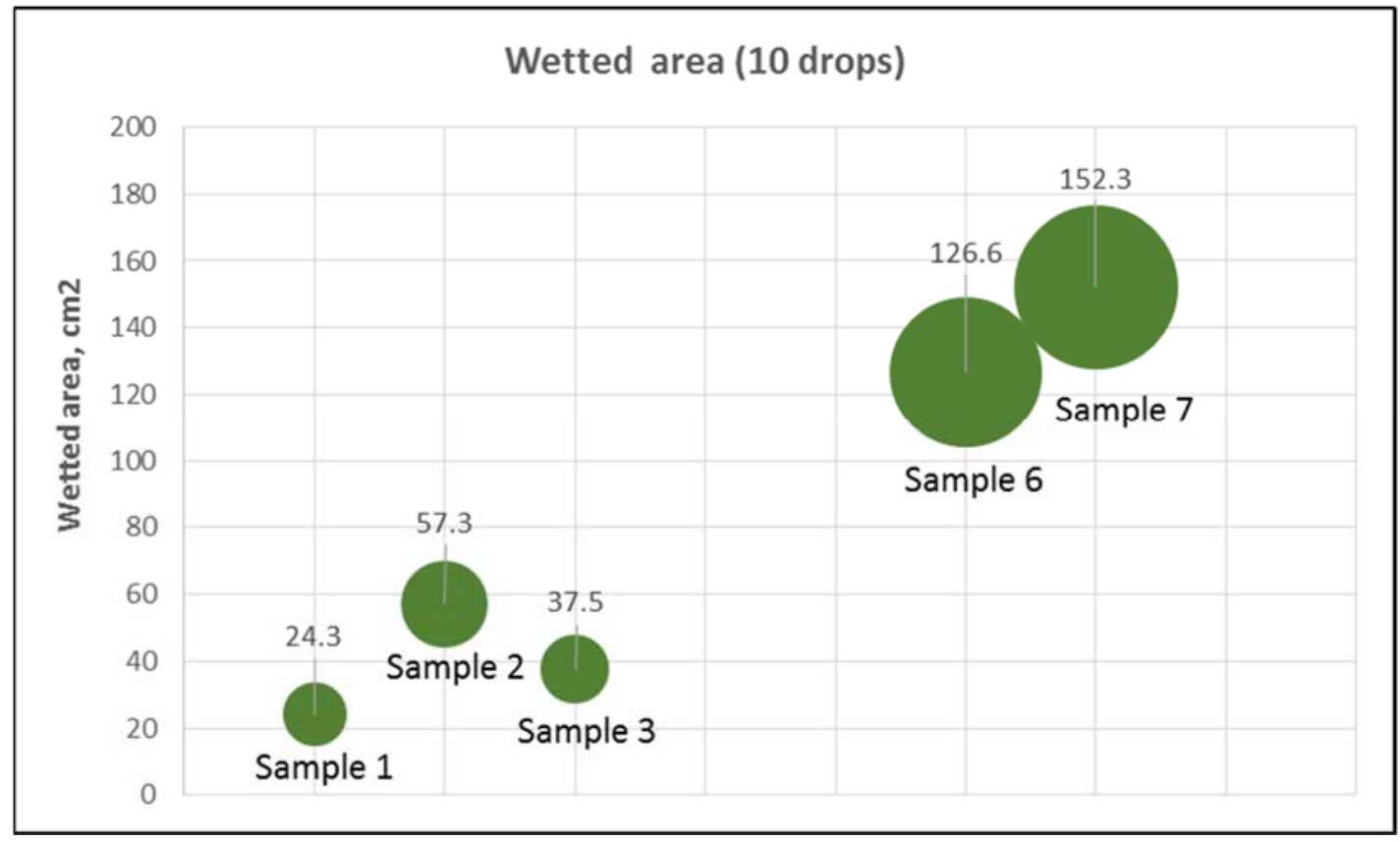

Figure 9 Wetted areas

Among the five fabrics Samples 6 and 7 presented outstanding general performance in terms of final wetted area $\left(126.6 \mathrm{~cm}^{2}\right.$ and $152.3 \mathrm{~cm}^{2}$ respectively), average diffusion rates $\left(0.506 \mathrm{~cm}^{2} / \mathrm{s}\right.$ and $0.406 \mathrm{~cm}^{2} / \mathrm{s}$ respectively), and also average evaporation rates, $(0.316 \mathrm{mg} / \mathrm{s}$ and $0 . \mathrm{mg} / \mathrm{s}$ respectively). However, further studies on their performance when they were adhered onto a heat transfer sheet (aluminium) using a heat transfer adhesive found Samples 6 and 7 became after being wetted, (see Figure 12), which could affect heat transfer efficiency within the evaporative cooler. This may be caused by their thin fabric thickness, which makes them difficult to be adhered evenly using a very thin layer of adhesive. Usually the wet surface material is attached to a heat transfer plate/sheet to form the wet side or evaporation side of the indirect evaporative cooling, as shown in Figure 1 (b), and when constructing a indirect evaporative cooler, wet surfaces of the two adjacent plates/sheets are against each other to build a wet channel. Similarly, the dry surfaces of two adjacent plates/sheets are against each other to build a dry channel. Although the above adhesive method is difficult to realize for the thin 
materials such as Samples 6 and 7, other methods, such as stretching the material against the heat transfer sheet by fixing its edges, could be applied to allow these materials to be used as a high-performance wet surface.

As shown in Figures 9 to 11, within the Samples 1, 2, and 3, Sample 2, i.e. the Kraft paper, gave the lowest evaporation rate of $0.179 \mathrm{mg} / \mathrm{s}$ and the lowest diffusion rate of $0.102 \mathrm{~cm}^{2} / \mathrm{s}$, although it had slightly larger final wetted area of $57.3 \mathrm{~cm}^{2}$, obtained by 10 drops of water (total $0.5 \mathrm{ml}$ ), due to it being the thinnest material among the three fabrics. In practice, usually a much higher density of water drops are continuously sprayed onto the wet surface by a water distributor in the indirect evaporative cooling system, which would provide the sufficient wetted area even the fabric's final wetted area conducted in this test is smaller. Therefore, the difference between the final wetted area among the three fabrics is less important than diffusion rate and evaporation rate. A higher diffusion rate can prevent a thin water film formation on the wet surface, the latter being detrimental to water evaporation and clearly higher evaporation rates can, no doubt, provide a higher cooling efficiency. Therefore, Samples 1 and 3, which have relatively higher diffusion rates of $0.116 \mathrm{~cm}^{2} / \mathrm{s}$ and $0.140 \mathrm{~cm}^{2} / \mathrm{s}$ respectively and evaporation rates of $0.215 \mathrm{mg} / \mathrm{s}$ and $0.204 \mathrm{mg} / \mathrm{s}$ respectively, are considered to have general better performance than Sample 2, i.e, the Kraft paper. It was found that Samples 1, 2 and 3 demonstrate good mechanical performance when they were adhered onto an aluminium heat transfer sheet using a heat transfer adhesive. 


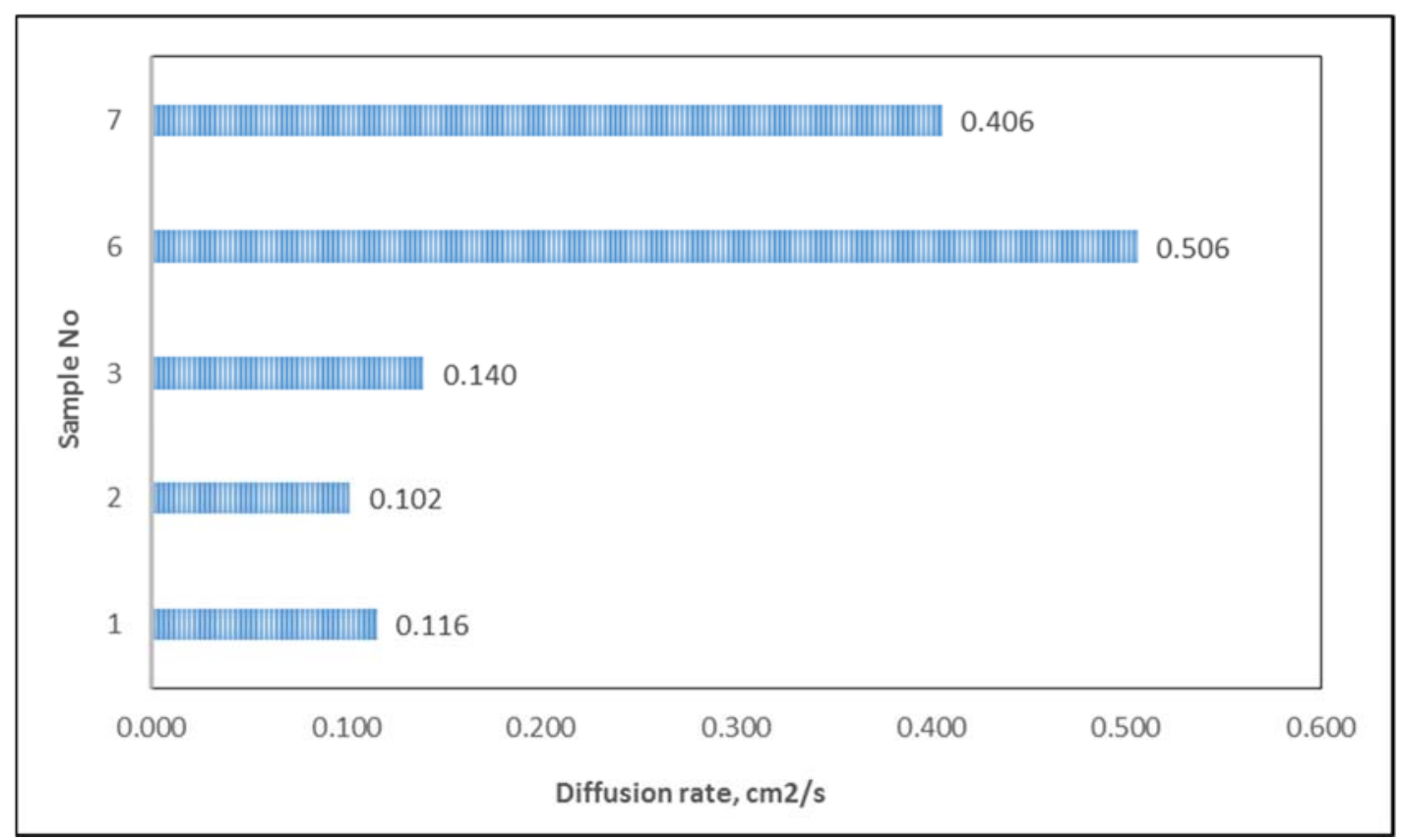

Figure 10 Diffusion rates

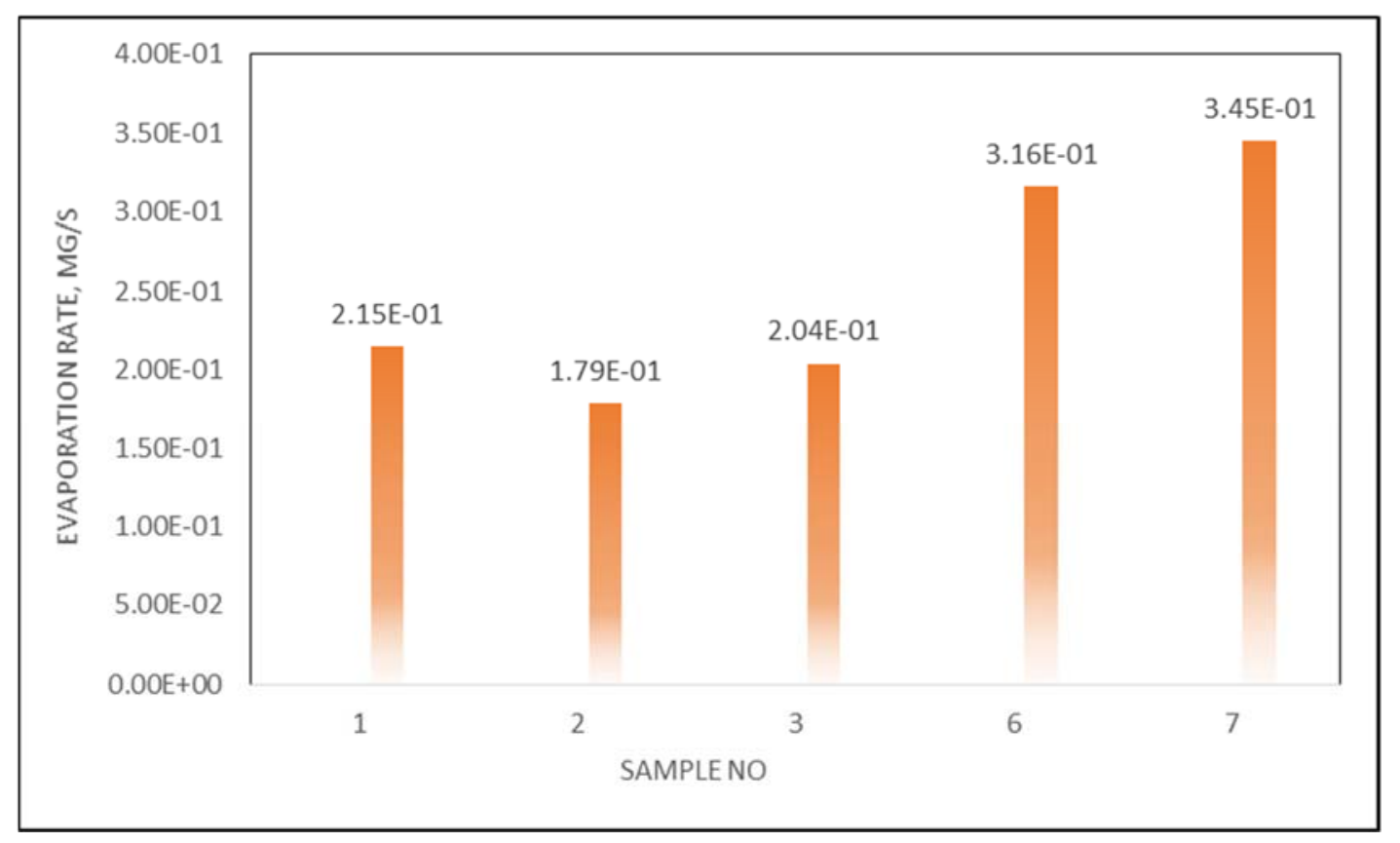

Figure 11 Evaporation rates 


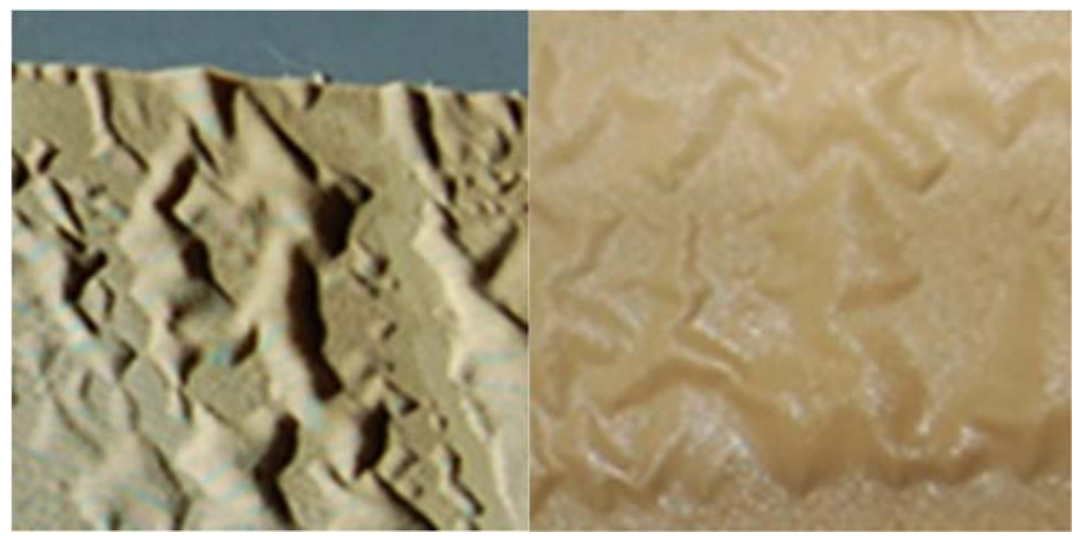

Figure 12. Images of distorted fabrics

\subsection{Evaluation of results from the tested fabrics}

Based on the above experimental work, a summary of the performance of the tested fabrics is shown in Table 5.

Table 5 A summary of the performance of the tested fabrics

\begin{tabular}{|c|c|c|c|c|c|}
\hline \multirow{2}{*}{$\begin{array}{l}\text { Sample } \\
\text { No }\end{array}$} & \multicolumn{4}{|c|}{ Performance } & \multirow[t]{2}{*}{ Suitability } \\
\hline & $\begin{array}{l}\text { Vertical } \\
\text { wicking }\end{array}$ & Diffusion & Evaporation & Mechanical & \\
\hline 1 & higher & higher & higher & good & $\begin{array}{l}\text { suitable with } \\
\text { higher } \\
\text { performance }\end{array}$ \\
\hline 2 & lowest & lowest & lowest & good & $\begin{array}{l}\text { suitable with } \\
\text { lower } \\
\text { performance }\end{array}$ \\
\hline 3 & higher & higher & higher & good & $\begin{array}{l}\text { suitable with } \\
\text { higher } \\
\text { performance }\end{array}$ \\
\hline 4 & higher & $\begin{array}{l}\text { Immediate } \\
\text { natural } \\
\text { diffusion is } \\
\text { not good }\end{array}$ & & & not suitable \\
\hline 5 & lower & $\begin{array}{l}\text { Immediate } \\
\text { natural } \\
\text { diffusion is } \\
\text { not good }\end{array}$ & & & not suitable \\
\hline 6 & lower & $\begin{array}{l}\text { highest } \\
\text { diffusion }\end{array}$ & $\begin{array}{l}\text { highest } \\
\text { evaporation }\end{array}$ & $\begin{array}{l}\text { Adhered } \\
\text { surface } \\
\text { distorted }\end{array}$ & $\begin{array}{l}\text { Suitable with } \\
\text { higher } \\
\text { performance } \\
\text { (not good for }\end{array}$ \\
\hline
\end{tabular}




\begin{tabular}{|l|l|l|l|l|l|}
\hline 7 & & & & & adhesive) \\
\hline 7 & lower & $\begin{array}{l}\text { highest } \\
\text { diffusion }\end{array}$ & $\begin{array}{l}\text { highest } \\
\text { evaporation }\end{array}$ & $\begin{array}{l}\text { Adhered } \\
\text { surface } \\
\text { distorted }\end{array}$ & $\begin{array}{l}\text { Suitable with } \\
\text { higher } \\
\text { performance } \\
\text { (not good for } \\
\text { adhesive) }\end{array}$ \\
\hline
\end{tabular}

As shown in Table 5, Samples 4 and 5 are not good choices for use as wet media within evaporative coolers as their immediate natural diffusion characteristics are not good. Samples 6 and 7 are too thin to allow simple adhesive bonding to a support heat transfer sheet, but other suitable attachment methods might be applied. Except for Samples 4 and 5, other Samples (1, 3, 6 and 7) showed better performance regarding to their wicking, diffusion and evaporation ability, compared to the Kraft paper. The results showed that the cloth fabrics, i.e. Samples 1, 3, 6 and 7, have great potential in terms of achieving more efficient indirect evaporative coolers. The percentage increase in fabric performance compared to Kraft paper is shown in Table 6.

Table 6 Percentage increase in performance relative to Kraft paper

\begin{tabular}{|l|l|l|l|}
\hline Performance & Vertical wicking rate, \% & Diffusion rate, \% & Evaporation rate, \% \\
\hline Sample 1 & 182 & 14 & 20 \\
\hline Sample 3 & 171 & 37 & 14 \\
\hline Sample 6 & 24 & 396 & 77 \\
\hline Sample 7 & 7 & 298 & 93 \\
\hline
\end{tabular}

For a large scale indirect evaporative cooler, appropriate fabric adhered to rigid supportive sheet has been found to be the most effective and simple way to construct a wet channel wall for indirective evaporative coolers. For this reason, it is considered that Samples 1 and 3 are the most suitable materials for this application. 


\section{CONCLUSIONS}

Seven different samples including six cloth fabrics and one of Kraft paper were experimentally studied in relation to their performance for indirect evaporative cooling applications. Tests were carried out to investigate their wicking ability, wetted areas, diffusion rates and evaporation rates. The results of the cloth fabrics have been analysed and compared to that of conventionally used Kraft paper. It was found that four of the fabrics have superior properties in terms of moisture wicking ability, diffusivity and evaporation ability compared to that of conventionally used Kraft paper. Two of them have low immidiate diffusion ability and were considered not suitable for evaporative cooling applications. A potential mechanical problem of the fabrics for wet surface applications, i.e, wet fabric distorted when adhered to the support sheet, has been identified during the study. A general assessment concerning both the moisture transfer and mechanical properties (ease of adhesive bonding) found that Coopass ${ }^{\circledR}$ bird eye mesh fabric and Bamboo charcoal ${ }^{\circledR}+$ Coolmax active fabric are most suitable for indirect evaporative cooling applications. The two fabrics have increased vertical wicking rates of $182 \%$ and $171 \%$, diffusion rates of $14 \%$ and $37 \%$ and evaporation rates of $20 \%$ and $14 \%$ respectively, compared to the conventional Kraft paper. It was noticed the cost of Coopass ${ }^{\circledR}$ bird eye mesh fabric is much lower than Bamboo charcoal ${ }^{\circledR}+$ Coolmax active fabric, and therefore it will be the best choice for evaporative cooling applications. Use of these fabrics therefore offer great potential to enhance the efficiency of indirect evaporative cooling systems.

\section{Acknowledgement}

The authors would like to acknowledge sincere appreciation of financial support from the University of Hull, Engineering and Physical Sciences Research Council (EPSRC), Innovate 
UK and the Ministry of Science and Technology of China (EP/M507830/1).

\section{REFERENCES}

[1] R. Boukhanouf, H. G. Ibrahim, A. Alharbi, and M. Kanzari, Investigation of an Evaporative Cooler for Buildings in Hot and Dry Climates, Journal of Clean Energy Technologies, Vol. 2, No. 3, July 2014.

[2] Zhiyin Duan, Changhong Zhan, Xudong Zhao, Xuelin Dong. Experimental study of a counter-flow regenerative evaporative cooler. Building and Environment Vol 104 (2016) 47-58. [3] Changhong Zhan, Xudong Zhao, Stefan Smith, S.B.Riffat. Numerical study of a M-cycle cross-flow heat and exchanger for indirect evaporative cooling, Vol46, Issue 3 (2011), 657-668.

[4] Lertsatitthanakorn C, Rerngwongwitaya S, Soponronnarit S. Field. Experiments and Economic Evaluation of an Evaporative Cooling System in a Silkworm Rearing House. Biosystems Engineering 2006; 93(2):213-9.

[5] T. Ravi Kiran, S.P.S. Rajput. Cooling Capacity and Energy Saving Potential of Dew Point Evaporative Cooling System for Indian Buildings, International Journal of Renewable Energy Research, Vol 3, No1, 2013.

[6] Peng Xu, Xiaoli Ma, Diallo Thierno M. O., Xudong Zhao , Kevin Fancey, Deying Li, Hongbing Chen, Numerical Investigation of the Energy Performance of a Guideless Irregular Heat and Mass Exchanger with Corrugated Heat Transfer Surface for Dew Point Cooling, Energy 109 (2016)803-817. 
[7] J. Lin, K. Thu, T.D. Bui, R.Z. Wang, K.C. Ng, K.J. Chua. Study on dew point evaporative cooling system with counter-flow configuration, Energy Conversion and Management 109 (2016) 153-165.

[8] Bruno F. On-site experimental testing of a novel dew point evaporative cooler. Energy and Buildings 2011;43:3475-83.

[9] Velasco Gomez E, Tejero Gonzalez A, Rey Martınez FJ. Experimental characterisationof an indirect evaporative cooling prototype in two operating modes. Applied Energy 2012; 97(0):340-6.

[10] Zhao X, Liu Shuli, Riffat S.B. Comparative study of heat and mass exchanging materials for indirect evaporative cooling systems. Building and Environment 43 (2008) 1902-1911.

[11] Rajesh Maurya, Nitin Shrivastava, Vipin Shrivastava. Performance Evaluation of Alternative Evaporative Cooling Media, International Journal of Scientific \& Engineering Research, Volume 5, Issue 10, October-2014.

[12] Kulkarni R. K. and Rajput S. P. S. Comparative Performance Analysis of Evaporative Cooling Pads ofAlternative Configurations and materials, International Journal of Advances in Engineering \& Technology, Sept. 2013. ISSN: 22311963.

[13] Nitipong Soponpongpipat and Sukum Kositchaimongkol. Recycled High-Density Polyethylene and Rice Husk as a Wetted Pad in Evaporative Cooling System. American Journal of Applied Sciences 8 (2): 186-191, 2011.

[14] Faleh A Al-Sulaiman. Evaluation of the performance of local fibers in evaporative cooling. Energy Conversion and Management 43(16):2267-2273, November 2002.

[15] Banyat Niyomvasa and Bunjerd Potakarat. Performance Study of Cooling Pads, Advanced 
Materials Research Vol. 664 (2013) pp 931-935.

[16]Arobindo Chatterjee and Pratibha Singh, Studies on Wicking Behaviour of Polyester Fabric, Journal of Textiles, Volume 2014, Article ID 379731.

[17] Chunhong Zhu a, Masayuki Takatera, Effect of Fabric Structure and Yarn on Capillary Liquid Flow within Fabrics, Journal of Fiber Bioengineering and Informatics 6:2 (2013) 205215.

[18] D. Knittel and E. Schollmeyer, Notes on future developments for textile finishing processes, The Journal of The TextileInstitute, vol. 91, part 3, pp. 166-167, 2000.

[19] R. Fangueiro, A. Filgueiras and F. Soutinho. Wicking Behavior and Drying Capability of Functional Knitted Fabrics, Textile Research Journal, March 18, 2010.(doi:10.1177/0040517510361796).

[20] S. C. Amico and C. Lekakou. Mathematical modelling of capillary micro-flow through woven fabrics, Composites A, vol. 31, no. 12, pp. 1331-1344, 2000.

[21] S. C. Amico and C. Lekakou. Axial impregnation of a fiber bundle-part 1: capillary experiments, Polymer Composites, vol. 23, no. 2, pp. 249-263, 2002.

[22] S. C. Amico and C. Lekakou, Axial impregnation of a fiber bundle-part 2: theoretical analysis," Polymer Composites, vol.23, no. 2, pp. 264-273, 2002.

[23] P. K. Chatterjee, Absorbent Technology, Elsevier Scientific, New York, NY, USA, 1985.

[24]Y. K. Kamath, S. B. Hornby, H. D.Weigmann, and M. F.Wilde, Wicking of spin finishes and related liquids into continuous filament yarns, Textile Research Journal, vol. 64, no. 1, pp. 33-40, 1994. 
[25] A. B. Nyoni and D. Brook, "Wicking mechanisms in yarns - the key to fabric wicking performance," Journal of the Textile Institute, vol. 97, no. 2, pp. 119-128, 2006.

[26] A. Perwuelz, P. Mondon, and C. Caze, "Experimental study of capillary flow in yarns," Textile Research Journal, vol. 70, no. 4,pp. 333-339, 2000.

[27] A. Perwuelz, M. Casetta, and C. Caze, "Liquid organisation during capillary rise in yarnsinfluence of yarn torsion," Polymer Testing, vol. 20, no. 5, pp. 553-561, 2001.

[28] E. W. Washburn, "The dynamics of capillary flow," Physical Review, vol. 17, no. 3, pp. 273-283, 1921.

[29] T. Liu, K.-F. Choi, and Y. Li, “Wicking in twisted yarns," Journal of Colloid and Interface Science, vol. 318, no. 1, pp. 134-139, 2008.

[30] Kissa E. Capillary sorption in fibrous assemblies. J Colloid Interf Sci 1981; 83(1): 265272.

[31] Craig Burton Smile. Critical evaluation of wicking in performance fabrics, MSc thesis, Georgia Institute of Technology, USA, December 2004.

[32] D Raja1, G Ramakrishnan, V Ramesh Babu, M Senthilkumar and MB Sampath. Comparison of different methods to measure the transverse wicking behaviour of fabrics. Journal of industrial textiles, 2014, Vol 43(3) 366-382. 